\title{
Mechanical properties of high-density TRIP steel honeycomb structures with varying cell profiles under different loading conditions
}

\author{
Christine Baumgart ${ }^{1 *}$, Christian Weigelt ${ }^{2}$, Christos G. Aneziris ${ }^{2}$, and Lutz Krüger $^{1}$ \\ ${ }^{1}$ Institute of Materials Engineering, TU Bergakademie Freiberg, 09599 Freiberg, Germany \\ ${ }^{2}$ Institute of Ceramic, Glass and Construction Materials, TU Bergakademie Freiberg, 09599 Freiberg, Germany
}

\begin{abstract}
As the mechanical properties of honeycomb structures are influenced by several parameters, detailed analysis is necessary before their potential application in transportation industry components. Previous Finite Element Model (FEM)-based numerical analysis demonstrated that variation in cell geometry affects the achievable strength level and, thus, the energy absorption capability. According to this FEM study, the Kagome geometry - an ordered sequence of hexagons and triangles - exhibits properties that are particularly promising when compared to the square-celled structures investigated to date. When the load is applied parallel to the channel axis (the out-of-plane direction), the increment of strength is comparatively low, whereas in the in-plane direction (loading orthogonal to the channel axis), the dissipated specific energy can reach almost double that of the square-celled structure. In this study, the results of static and dynamic compression tests - performed in the out-of-plane and in-plane modes - are presented to examine the influence of strain rate and loading direction on the characteristic deformation stages of squarecelled and Kagome structures. Particular attention is paid to deformation induced martensite formation in the cell wall material, indicating the TRansformation Induced Plasticity (TRIP) effect as a function of applied cell geometry, strain rate and loading direction.
\end{abstract}

\section{Introduction}

Cellular structures are promising for application in construction elements of the automotive and aerospace industries due to their high stiffness and high strength-toweight ratio [1]. In principle, a classification can be made into three- and two-dimensional structures. While in three-dimensional structures, like foams, the polyhedra are arranged spatially, two-dimensional structures are characterized by a fixed arrangement of polygons in a plane which continues in a linear fashion in the third dimension [2]. Anisotropic mechanical properties are common for such two-dimensional structures. Higher strength levels and, thus, energy absorption capabilities can be obtained in the out-ofplane direction than in the in-plane mode [3-5]. However, alteration of the cell shape can result in an improvement, as discussed in the literature for both twoand three-dimensional structures [6-7]. If the load is applied perpendicular to the channel axis, either cell-wall stretching or cell-wall bending becomes the dominant deformation mechanism. Cell-wall stretching is preferable, and occurs in equilateral triangular cells, Kagome cells and diamond-shaped cells (stiffened transversely) with rigid joints. Consequently, the collapse load depends mainly on the axial strength of the struts. In contrast, hexagonal cells or square-celled structures that are loaded diagonally to the cell wall orientation or exposed to shear forces are concerned mainly with bending-dominated mechanisms. This results in lower stiffness and collapse strength during deformation.

In order to enhance the mechanical properties, an additional approach is the alteration of the cell wall material, which can also be beneficial in the out-of-plane mode. Steels exhibiting the TRansformation Induced Plasticity (TRIP) effect would seem to provide an excellent option due to the simultaneous increase in strength and ductility associated with this effect. In principle, the martensitic phase transformation is triggered by exceeding the nucleation threshold at the martensite start temperature $\left(\mathrm{M}_{\mathrm{S}}\right)$. In TRIP steels, however, a certain amount of mechanical work can be added to the chemical driving force so that the transformation occurs above $M_{s}$ but below $M_{d}$, which describes the upper temperature limit $[8,9]$. On the other hand, whether $\varepsilon$ - or $\alpha^{\prime}$-martensite formation becomes the dominant deformation mechanism depends on both the chemical driving force and the stacking fault energy. It has been shown that in $\mathrm{CrMnNi}$ steels with stacking fault energies of over $\sim 40 \mathrm{~mJ} / \mathrm{m}^{2}$, dislocation glide is prevalent, whereas between 20 and $40 \mathrm{~mJ} / \mathrm{m}^{2}$, deformation twinning occurs. For energies below $\sim 20 \mathrm{~mJ} / \mathrm{m}^{2}$, a continuous transition to $\varepsilon$ - and $\alpha^{\prime}-$ martensite formation takes place. However, it has to be 
taken into account that the stacking fault energy is strongly influenced by the temperature, which also influences the chemical driving force [10-11]. Hence, if the material is subjected to an increased strain rate where a high amount of mechanical work is transformed into thermal energy by friction processes, the martensitic transformation will be suppressed [12-13].

The focus of this study is the comparative investigation of two honeycomb structures with Kagome and square-celled profiles loaded in the out-of-plane and in-plane directions in order to determine the influence of cell structure on the compressive deformation and failure behaviour. Additionally, the occurrence of the TRIP effect in the CrMnNi steel cell wall material is discussed as a function of the cell geometry, the strain rate applied and the direction of loading. The evaluation is conducted by ferromagnetic phase measurement: therefore, only ferromagnetic $\alpha$ '-martensite is considered.

\section{Manufacturing and mechanical testing}

A powder metallurgical process was used to manufacture the honeycomb structures [14]. To this end, a gasatomized steel powder $\left(\mathrm{d}_{90}=33 \mu \mathrm{m}\right)$ was blended with an organic binder system, plasticizers and deionized water. Table 1 describes the chemical composition of the powder and indicates the main alloying elements - i.e. chrome, manganese and nickel - with the abbreviation 17-7-6. This alloying system was selected so that the occurrence of deformation induced martensite formation (the TRIP effect) could be ensured at room temperature.

In the subsequent manufacturing step, the resultant shapeable mass is pressed through a single-screw extruder with a replaceable die at the outlet. In this way, different cellular structures can be produced. For the investigated structures, a square external contour was used with sides measuring $25 \times 25 \mathrm{~mm}$. In order to maintain the relative density of the honeycombs at a comparable level, the width of the cell walls was varied between the Kagome $(400 \mu \mathrm{m})$ and square-celled structures $(350 \mu \mathrm{m})$. The strands produced were cut into near-sample dimensions before the binder was burned out in a furnace (normal air atmosphere) at $450{ }^{\circ} \mathrm{C}$. In the final processing step, sintering of the brown bodies is carried out at $1350{ }^{\circ} \mathrm{C}$ for $2 \mathrm{~h}$ in an argon atmosphere.

Table 1. Chemical composition of initial steel powder and sintered honeycomb structures.

\begin{tabular}{|c|c|c|c|c|c|c|c|}
\hline & $\mathrm{C}$ & $\mathrm{Cr}$ & $\mathrm{Mn}$ & $\mathrm{Ni}$ & $\mathrm{Si}$ & $\mathrm{N}$ & $\begin{array}{c}\mathrm{Fe}+ \\
\text { others }\end{array}$ \\
\hline $\begin{array}{c}\text { 17-7-6 } \\
\text { powder }\end{array}$ & 0.03 & 17.0 & 6.5 & 6.1 & 0.3 & 0.08 & bal. \\
\hline Square & 0.04 & 17.1 & 5.9 & 6.2 & 0.3 & 0.03 & bal. \\
\hline Kagome & 0.05 & 17.0 & 5.9 & 6.2 & 0.3 & 0.03 & bal. \\
\hline
\end{tabular}

Prior to mechanical testing, the X1-X2 planes of the samples were machined in order to obtain a height of $22 \mathrm{~mm}$ and to ensure perpendicularity with respect to the
X3 direction. For static compression testing, an MTS servo-hydraulic testing machine equipped with an extensometer was used. The adjusted velocity of the punch was $0.022 \mathrm{~mm} / \mathrm{s}$, effecting a strain rate of $0.001 \mathrm{~s}^{-1}$ in the samples. Dynamic testing was conducted by using a drop-weight tower with a magnetic displacement measurement system (Fritz Kübler $\mathrm{GmbH}$ ). The punch was fitted with strain gages to record the force signals. When testing in the out-of-plane compression mode, the weight (approx. $190 \mathrm{~kg}$ ) was released from a height of $800 \mathrm{~mm}$, whereas for in-plane compression, drop heights of $600 \mathrm{~mm}$ were used, generating velocities of $4 \mathrm{~m} / \mathrm{s}$ and $3.4 \mathrm{~m} / \mathrm{s}$, respectively.

For both deformation speeds and load directions, intermediate strain levels of approx. $10 \%, 20 \%$ and $35 \%$ engineering strain were set to measure the ferromagnetic phase fraction and, thus, the kinetics of the TRIP effect. To this end, slices with a thickness of $3.5 \mathrm{~mm}$ were cut from the centre of the samples and analysed in a MSAT magnetic saturation device (Metis Instrument and Equipment NV) equipped with a fluxmeter 480 (Lake Shore).

\section{Results and discussion}

Fig. 1 contains images of the cubic samples with squarecelled and Kagome profiles in the sintered state. In addition, magnified recordings of the cells are included that emphasize the decrease in cell-wall thickness induced by shrinkage during the sintering process. The thickness averaged $330 \mu \mathrm{m}$ for the Kagome structure, and $275 \mu \mathrm{m}$ for the square-celled structure. With respect to the chemical composition, only small alterations arose from the sintering process. As Table 1 reveals, the manganese content decreased by $0.6 \mathrm{wt} \%$ for both geometries, while the carbon and nickel concentrations increased slightly by $0.01-0.02 \mathrm{wt} \%$ and $0.1 \mathrm{wt} \%$, respectively. Nevertheless, the sintered honeycombs exhibited an austenitic matrix with a low amount of $\delta$ ferrite. Since $\delta$-ferrite is also ferromagnetic, the ferromagnetic contents measured and presented in the following sections have to be considered as aggregates of deformation induced $\alpha$-martensite and $\delta$-ferrite.

By comparing the profiles in Fig. 1, the anisotropy of the Kagome profile becomes obvious. For the out-ofplane compression tests where the load was applied in the X3 direction, the mechanical properties were not affected. However, when the load was applied in the inplane $(\mathrm{X} 1 / \mathrm{X} 2)$ direction, a distinction became necessary. In the following, the denotation 'Kagome' represents the orientation where the load was parallel to the X1 direction, and ' Kagome $90^{\circ}$ ' indicates load application in the $\mathrm{X} 2$ direction. 


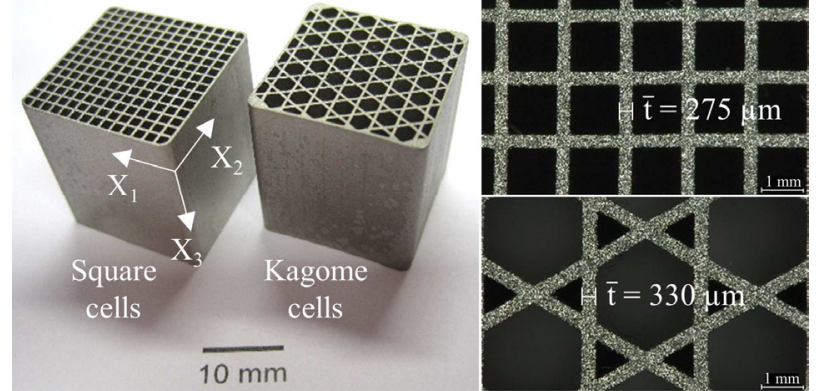

Fig. 1. Honeycomb structures with varying cell profiles in the sintered state.

\subsection{Out-of-plane compression}

The static and dynamic stress-strain curves of the Kagome and square-celled structures are displayed in Fig. 2. It should be note that the dynamic strain rate depicted refers to the region of stable deformation in the pre-buckling stage. In addition, the stress was normalized to the relative density of the structures in order to correct the influence of small differences in relative density and microporosity.
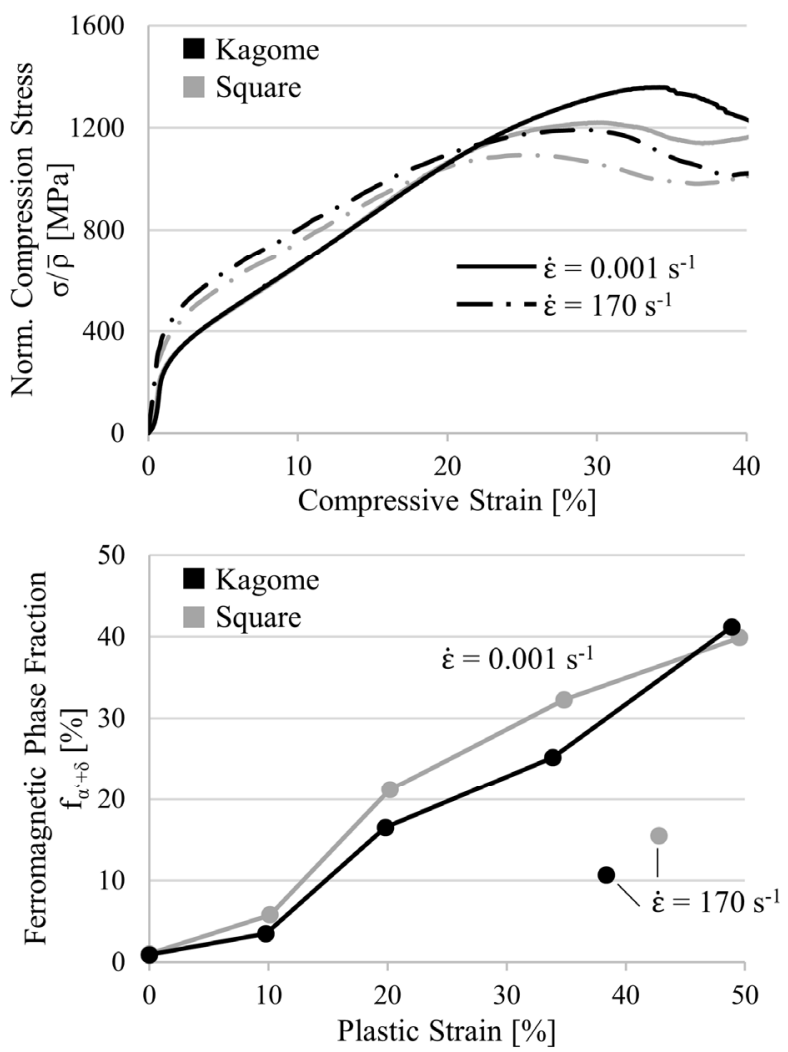

Fig. 2. Static and dynamic stress-strain curves normalized to the relative density and ferromagnetic phase fraction as a function of plastic strain of the Kagome and square-celled honeycomb structures recorded in the out-of-plane mode.

The curve progressions demonstrate that the linearelastic region and the attainable stress level in the prebuckling stage at low strains were almost unaffected by the cell geometry. Only a slight stress offset was evident between the Kagome and square-celled structures for dynamic strain rates, although the stress slope was comparable. However, by comparing the onset of the post-buckling stage, which is associated with structural bifurcation [15], lower strains and stresses were measurable for the square-celled structure. Similarly, the collapse strength was lower and collapse was initiated at lower stages of deformation. Hence, the area enclosed by the Kagome curves was greater than for the squarecelled structure, indicating a higher absorbed energy. This fact may also be understood by comparing the adjusted degrees of plastic deformation of the samples compressed dynamically. The energy content of the drop weight was sufficient to deform the square-celled structure by $43 \%$, whereas only $38 \%$ could be recorded for the Kagome sample.

In addition, Fig. 2 shows the ferromagnetic phase fraction as a function of plastic strain for both honeycomb structures. Samples deformed statically by $10 \%$ exhibited a comparable ferromagnetic phase fraction. At strains of $20 \%$ and $35 \%$, however, the amount of deformation induced $\alpha$-martensite was distinctly higher for the square-celled structure. One possible explanation was that of the different deformation stages that existed at the set strains. Since structural bifurcation is associated with a transition from almost homogenous, bending-dominated deformation to instable deformation with an inhomogeneous strain distribution, local areas existed that exhibited an enhanced TRIP effect [4]. As outlined above, the postbuckling stage in the Kagome structure was initiated at higher strains, implying a delayed increase in $\alpha$ martensite formation. A finer increment in deformation steps and ferromagnetic phase measuring would provide a reliable indication. However, it is also conceivable that the varying contents arose due to the difference in cell size (cell number) and cell wall thickness. At 50\% plastic strain, the differences adapted such that a ferromagnetic phase fraction of approx. 40\% was measurable in both samples. Considering the ferromagnetic contents of the dynamically deformed structures at $38 \%$ and $43 \%$ plastic strain, a distinctly lower value became obvious when compared to the samples strained statically to $35 \%$ and $50 \%$, respectively. By increasing the deformation speed, a forced distortion in deformation bands was involved. Hence, the probability of their intersection increased, as did the formation of $\alpha^{\prime}$-martensite nuclei. In addition, dislocation glide - which occurred in addition to the TRIP effect but was less dominant - was restricted due to the reduced waiting time of a dislocation before an obstacle, along with the lower probability of the emergence of a sufficient thermal fluctuation for overcoming such obstacles. Consequently, higher stresses were required for plastic flow, which was illustrated by the increased compressive yield strength and compressive stress in the pre-buckling stage of the dynamically deformed samples shown in Fig. 2. On the other hand, dynamic strain rates resulted in quasiadiabatic sample heating, which involved material softening [16]. Thus, the dynamic and static stress-strain curves intersected at elevated strains (Fig. 2), and the driving force for $\alpha$ '-martensite formation was reduced as confirmed by the ferromagnetic phase content. 


\subsection{In-plane compression}

The results of static in-plane deformation are included in Fig. 3. For the square-celled geometry, structural collapse was closely linked to the linear-elastic region, indicating the absence of a distinct pre-buckling stage. In contrast, a work hardening stage subsequent to the linear-elastic region was recordable for both Kagome orientations, whereby the extent was greater for the Kagome $90^{\circ}$ alignment. After structural collapse, the highest plateau strength was measurable for the Kagome $90^{\circ}$ direction. By comparing the ferromagnetic phase fraction, an increased value could be measured for the square-celled structure at $10 \%$. However, for deformations equal to or greater than $20 \%$ of plastic strain, the Kagome $90^{\circ}$ alignment contained the highest amount of $\alpha^{\prime}$-martensite. One explanation could be the macroscopic failure behaviour of the structures, which is shown e.g. in Fig. 4 for an engineering strain of 25\%.
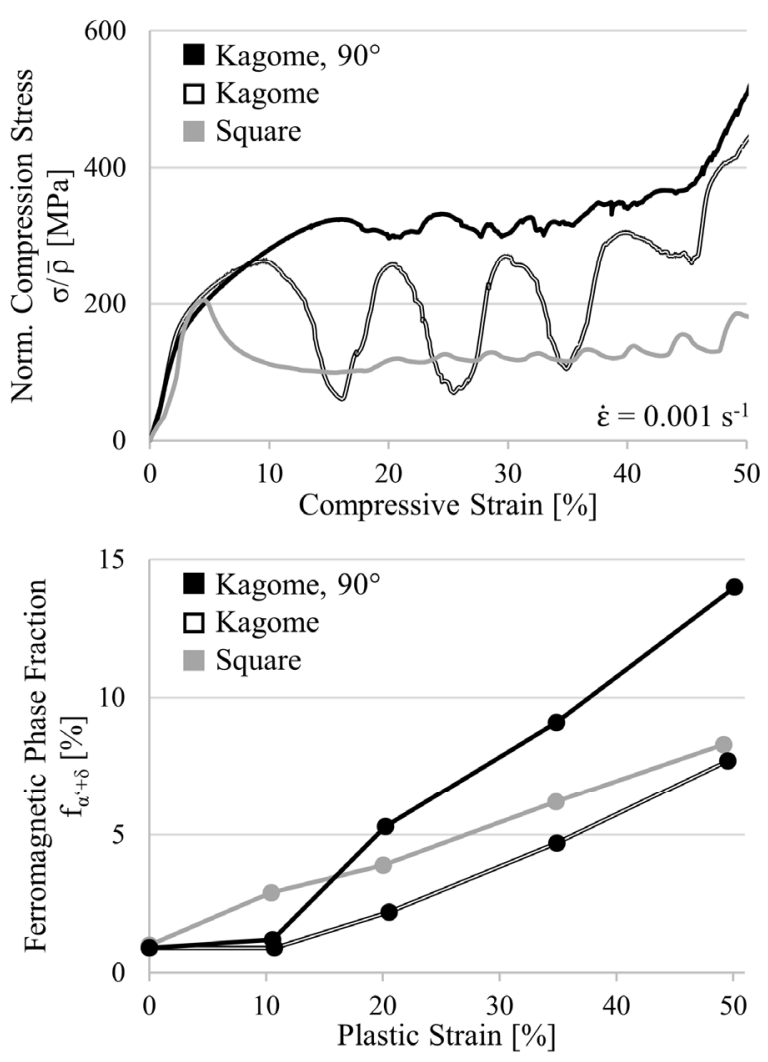

Fig. 3. Static stress-strain curves normalized to relative density and ferromagnetic phase fraction as a function of the plastic strain of Kagome, Kagome $90^{\circ}$ and square-celled honeycomb structures recorded in the in-plane mode.

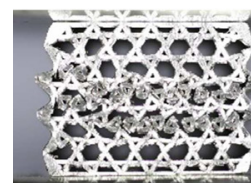

Kagome

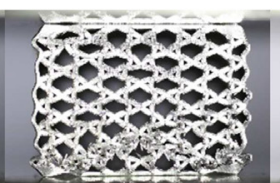

Kagome $90^{\circ}$

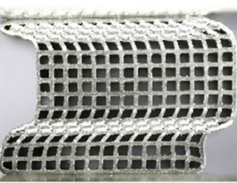

Square $\frac{10 \mathrm{~mm}}{}$
Fig. 4. Deformation patterns of Kagome, Kagome $90^{\circ}$ and square-celled structures at engineering strains of $25 \%$ for the in-plane mode and quasi-static strain rates.
In the Kagome direction, initial buckling occurred in the cell walls angled to the direction of loading, followed by a cell wall rotation process which led to the fracture of cell nodes. Fig. 4 illustrates that the deformation was concentrated in the centre of the sample. Although the vertically aligned cell walls of the Kagome $90^{\circ}$ orientation also experienced buckling and formed a triangle-shaped deformation band at the base of the samples, only a few cell nodes are fractured. Therefore, the forces applied could be distributed to the intact cell walls and, thus, $\alpha^{\prime}$-martensite formation could proceed.

Even at elevated strains where the number of fractured nodes increased, the fragments were well oriented and the strength of the cell walls without any cracks avoided a stress oscillation - as was recorded for the Kagome orientation. However, the back-scattered electron (BSE) images in Fig. 5, which were taken at $35 \%$ plastic strain, demonstrated that the deformation was not homogenous.

A strut angled with respect to the direction of loading (a) exhibited only occasional deformation bands, whereas an adjacent vertical cell wall (b) exhibited increased distortion fields with deformation bands containing $\alpha^{\prime}$-martensite nuclei. A magnified section is displayed in the upper right corner of Fig. 5 b) and is marked with a dashed frame. It should be noted that the dark microstructural components were oxidic compounds, which emerged during the powder metallurgical manufacturing process in both geometries.

Currently, several studies are being conducted with the aim of suppressing their formation. For the squarecelled structure, in-plane deformation resulted in a sequential failure of several cell planes (cf. Fig. 4), which is also observed in other studies for honeycomb structures with square and hexagonal cells [3, 17]. Therefore, a large sample area experienced less deformation, which restricted the occurrence of the TRIP effect.

Despite the Kagome honeycomb structure providing increased strength in the in-plane mode for both orientations, the results generated in the out-of-plane direction could not be matched due to constrained deformation in the X1-X2 plane. While the load was applied parallel to the X3 direction (i.e. out-of-plane) deformation took place in all three spatial directions.

Fig. 6 shows the dynamically recorded stress-strain curves in the in-plane direction. Due to the absence of an extended stable deformation stage, especially in the case of the square-celled structure, the average strain rate was calculated up to an engineering strain of $50 \%$. Similarly to the out-of-plane mode, an increase in strain rate resulted in an increase in compressive stress. It was evident that the stress signal in the plateau region at higher strains oscillated around an elevated average value, which was pronounced for the Kagome orientation and the square-celled structure. For Kagome $90^{\circ}$, a distinct enhancement did not arise. However, an increased oscillation was detectable, as was the case for the square-celled structure. 

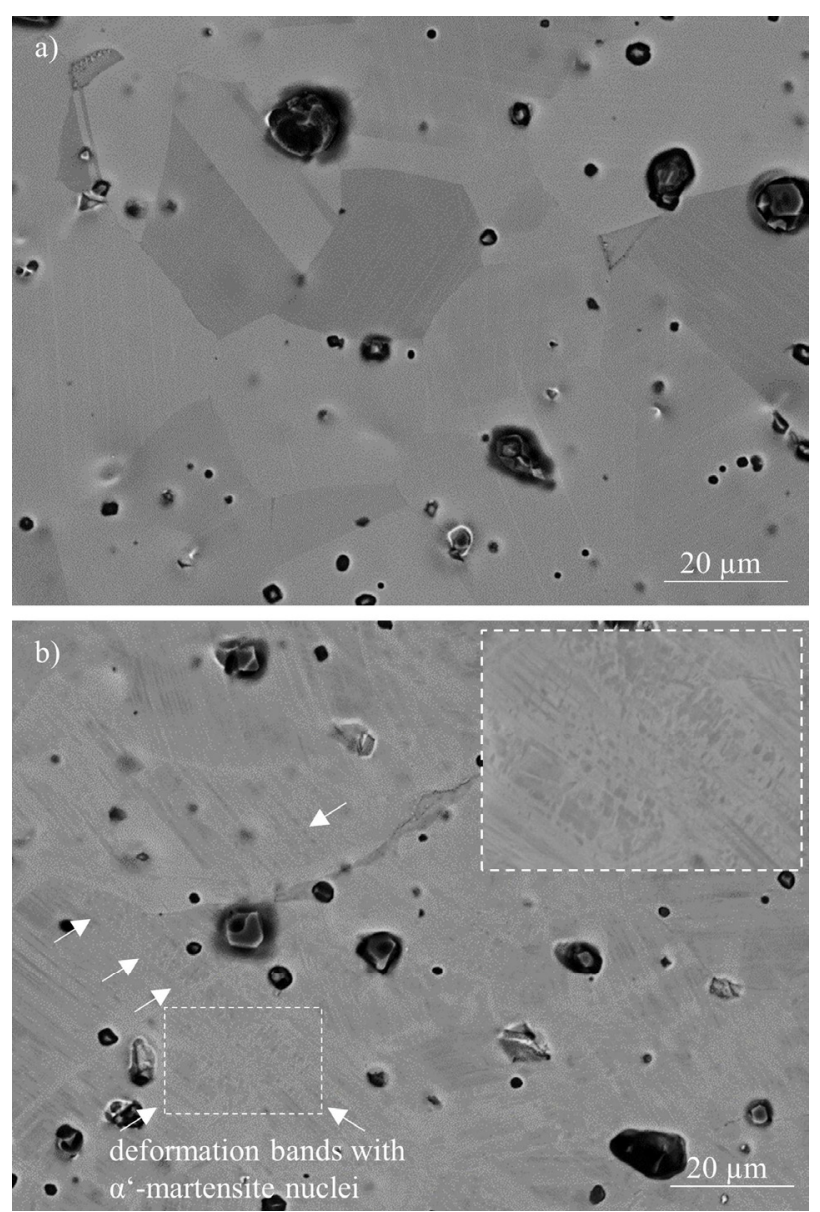

Fig. 5. BSE images of Kagome $90^{\circ}$ sample at a deformation degree of $35 \%$ : a) strut angled with respect to the direction of loading, b) vertical strut.

Quasi-adiabatic heating was also responsible for the decreased $\alpha^{\prime}$-martensite formation in this direction of loading. Between plastic strains of $10 \%$ and $35 \%$, the ferromagnetic content was not markedly different for the two Kagome orientations and the square-celled structure. In addition, there was no distinct growth in the absolute value. In order to form a comparable amount of $\alpha^{\prime}$ martensite, the dynamically deformed square-celled structure had to be strained to approx. $70 \%$, whereas under static load, conditions of approx. $50 \%$ were sufficient. As a result of the dynamic in-plane tests, it could be concluded that the Kagome $90^{\circ}$ orientation also provided the highest energy absorption capability in this direction of loading, followed by the Kagome alignment - a fact evident from the adjusted degrees of deformation. The energy content of the drop weight tower deformed the Kagome $90^{\circ}$ orientation with a plastic strain of $63 \%$, compared to $67 \%$ for the Kagome orientation and $71 \%$ for the square-celled structure.
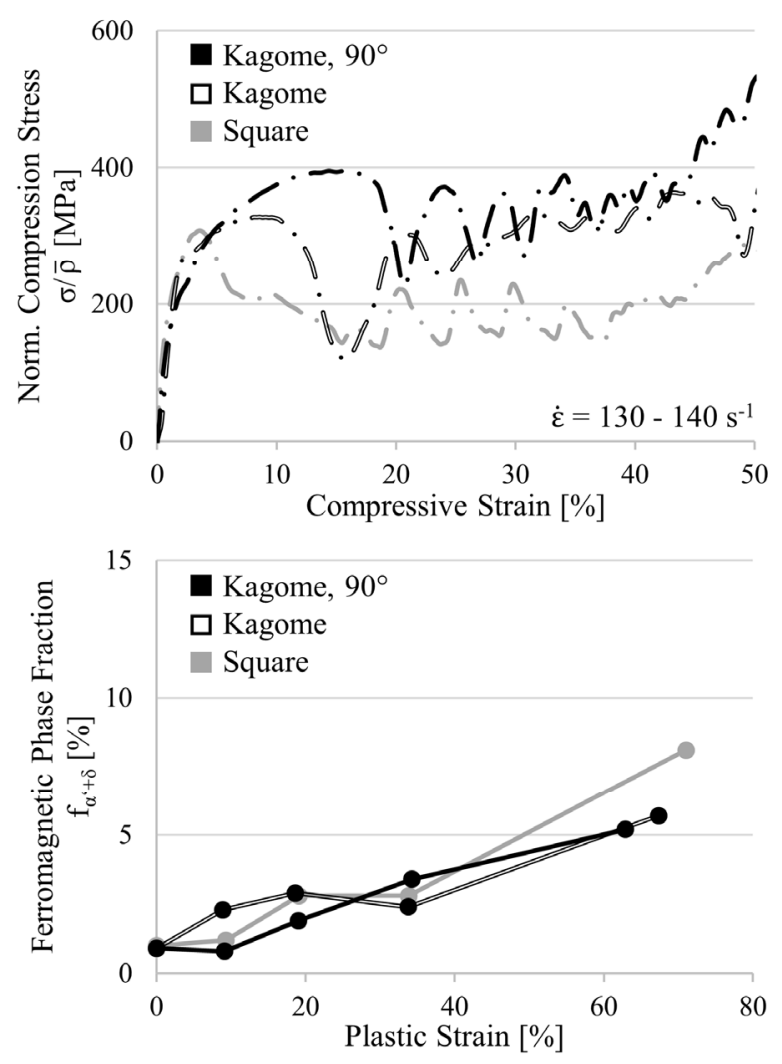

Fig. 6. Dynamic stress-strain curves normalized to relative density and ferromagnetic phase fraction as a function of the plastic strain of the Kagome, Kagome $90^{\circ}$ and square-celled honeycomb structures recorded in the in-plane mode.

\section{Conclusion}

Static and dynamic out-of-plane and in-plane compression tests, conducted on TRIP steel honeycomb structures with Kagome and square-celled structures produced the following results and findings:

\section{Out-of-plane loading}

- Linear-elastic regions and strength levels in the prebuckling stage at low deformation stages were almost unaffected by the cell geometry for both strain rates

- Earlier onset of the post-buckling stage in the square-celled structure may have resulted in different $\alpha^{\prime}$-martensite contents at $20 \%$ and $35 \%$ plastic strain in the statically deformed samples due to inhomogeneous strain distributions

- Dynamic strain rates led to an increase in compressive yield strength and compressive stress in the pre-buckling stage based on forced distortion in the deformation bands - which resulted in the promotion of $\alpha^{\prime}$-martensite formation - and the restriction of dislocation glide

- The intersection of dynamic and static stress-strain curves at elevated strains and the lower $\alpha^{\prime}$ - 
martensite content in the dynamically deformed samples resulted from quasi-adiabatic heating

\section{In-plane loading}

- A distinct work-hardening stage subsequent to the linear-elastic region was evident for both Kagome orientations, in contrast to the square-celled structure

- The highest plateau strength and the greatest amount of $\alpha^{\prime}$-martensite ( $\geq 20 \%$ plastic strain) was recorded for the Kagome $90^{\circ}$ alignment in the statically deformed samples

- As in the out-of-plane mode, an increase in strain rate led to an increase in compressive stress, which continued for the square-celled structure and the Kagome orientation up to the plateau region

- No appreciable difference in $\alpha^{\prime}$-martensite formation was evident due to dynamic deformation for all samples

Finally, it can be concluded that the substitution of square cells by Kagome cells resulted in beneficial mechanical properties for both the out-of-plane and inplane conditions of loading as well as at various strain rates. In the in-plane mode, in particular, a greater area was enclosed by the stress-strain curves of the Kagome honeycomb structures, implying a greater degree of energy absorption capability - which helped to close the still existing disparity with the out-of-plane properties.

The authors thank the German Research Foundation (DFG) for its financial support of the subproject B2 I "Experimental and model-based characterisation of strength, deformation and damage behaviour of optimized TRIP-steel modifications and TRIP-Matrix-Composites" as part of the "TRIP-MatrixComposite" Collaborative Research Centre (CRC 799) in which the presented results were obtained.

\section{References}

1. J. Banhart, Prog. Mater Sci. 46, 559-632 (2001)

2. L.J. Gibson, M.F. Ashby, Cellular Solids - Structure and Properties, Cambridge University Press, $2^{\text {nd }}$ ed. (1997)

3. A.M. Hayes, A. Wang, B.M. Dempsey, D.L. McDowell, Mech. Mater. 36, 691-713 (2004)

4. D. Ehinger, Dissertation, Institute of Materials Engineering, TU Bergakademie Freiberg (2013)

5. M.K. Kahn, T. Baig, S. Mirza, Mater. Sci. Eng., A 539, 135-142 (2012)

6. A.-J. Wang, D.L. McDowell, Int. J. Plast. 21, 285-320 (2005)

7. V.S. Deshpande, M.F. Ashby, N.A. Fleck, Acta Mater. 49, 1035-1040 (2001)

8. V.F. Zackay, E.R. Parker, D. Fahr, R. Busch, Trans. Am. Soc. Metals 60, 252- 259 (1967)

9. I. Tamura, Met. Sci. 16, 245-253 (1982)

10. S. Martin, S. Wolf, U. Martin, L. Krüger, Solid State Phenom. 172-174, 172-177 (2011)

11. S. Martin, S. Wolf, U. Martin, L. Krüger, D. Rafaja, Metall. Mater. Trans. A 47, 49-58 (2016)

12. J. Talonen, P. Nenonen, G. Pape, H. Hänninen, Metall. Mater. Transactions A 36A, 421-432 (2005)

13. S. Wolf, S. Martin, L. Krüger, U. Martin, Mater. Sci. Eng., A 594, 72-81 (2014)

14. C. Weigelt, C.G. Aneziris, A. Yanina, S. Guk, Steel Res. Int. 82 (9), 1080-1086 (2011)

15. D. Ehinger, L. Krüger, S. Krause, U. Martin, C. Weigelt, C. G. Aneziris, EPJ Web of Conferences 26, 01056 (2012)

16. D. Ehinger, L. Krüger, U. Martin, C. Weigelt, C.G. Aneziris, Int. J. Solids Struct. 66, 207-217 (2015)

17. S.D. Papka, S. Kyriakides, J. Mech. Phys. Solids 42 , 1499-1532 (1994) 